\title{
Trypophobia as an urbanized emotion: Comparative research in ethnic minority regions of China
}

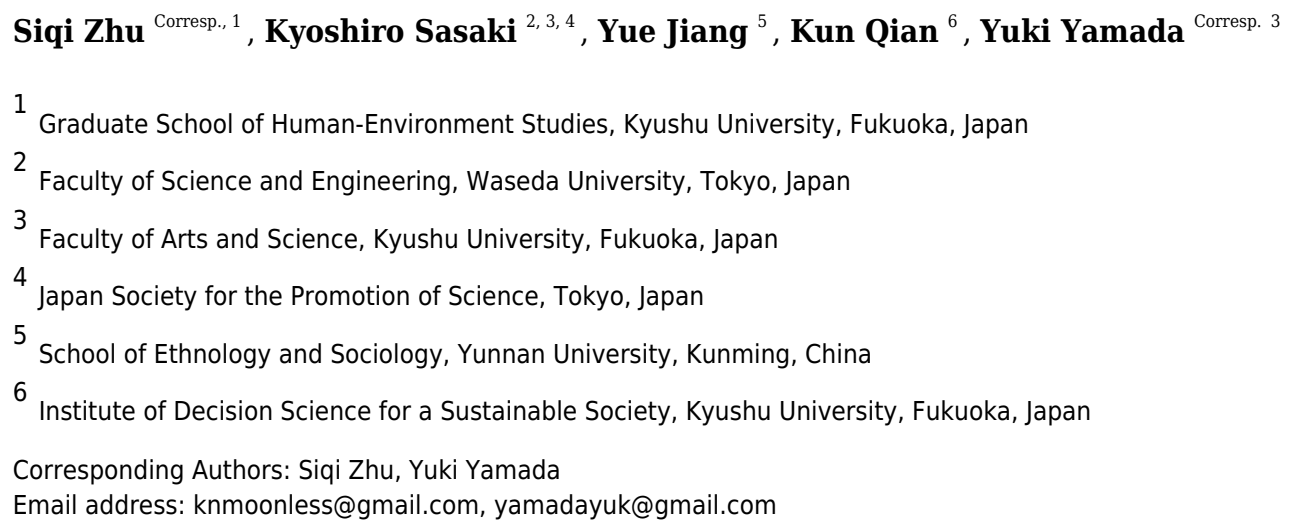

Trypophobia is a strong emotion of disgust evoked by clusters of holes or round objects (e.g., lotus seed pod). It has become increasingly popular and been studied since 2010s, mainly in the West and Japan. Considering this, trypophobia might be a modern emotion and hence, urbanization possibly plays key roles in trypophobia. To address this issue, we compared the degree of trypophobia between urban and less urban people in China. In an experiment, we asked participants about their degree of discomfort from trypophobic images. The results showed that trypophobia occurred in both groups, although the effect size was larger in urban than less urban people. Moreover, post-experimental interviews and post-hoc analyses revealed that older people in less urban area did not experience as much trypophobia. Our findings suggest that trypophobia links to urbanization and agerelated properties. 


\section{Trypophobia as an urbanized emotion: Comparative research in}

\section{2 ethnic minority regions of China}

\section{3}

4
Siqi Zhu ${ }^{1^{*}}$, Kyoshiro Sasaki2,3,4, Yue Jiang ${ }^{5}$, Kun Qian $^{6}$, and Yuki Yamada ${ }^{2 *}$

${ }^{1}$ Graduate School of Human-Environment Studies, Kyushu University, Fukuoka, Japan

${ }^{2}$ Faculty of Arts and Science, Kyushu University, Fukuoka, Japan

${ }^{3}$ Faculty of Science and Engineering, Waseda University, Tokyo, Japan

${ }^{4}$ Japan Society for the Promotion of Science, Tokyo, Japan

${ }^{5}$ School of Ethnology and Sociology, Yunnan University, Kunming, China

${ }^{6}$ Institute of Decision Science for a Sustainable Society, Kyushu University, Fukuoka, Japan

Word count: 3068

*Corresponding author 1: Siqi Zhu

Address: Graduate School of Human-Environment Studies, Kyushu University,

744 Motooka, Nishi-ku, Fukuoka 819-0395, Japan

E-mail: knmoonless@gmail.com

**Corresponding author 2: Yuki Yamada

Address: Faculty of Arts and Science, Kyushu University,

744 Motooka, Nishi-ku, Fukuoka 819-0395, Japan

E-mail: yamadayuk@gmail.com 
24 Trypophobia is a strong emotion of disgust evoked by clusters of holes or round objects (e.g., 25 lotus seed pod). It has become increasingly popular and been studied since 2010 s, mainly in the 26 West and Japan. Considering this, trypophobia might be a modern emotion and hence, 27 urbanization possibly plays key roles in trypophobia. To address this issue, we compared the 28 degree of trypophobia between urban and less urban people in China. In an experiment, we asked 29 participants about their degree of discomfort from trypophobic images. The results showed that 30 trypophobia occurred in both groups, although the effect size was larger in urban than less urban 31 people. Moreover, post-experimental interviews and post-hoc analyses revealed that older people 32 in less urban area did not experience as much trypophobia. Our findings suggest that trypophobia 33 links to urbanization and age-related properties. 


\section{Introduction}

38 Clusters of holes or round objects can induce strong feelings of disgust. This phenomenon is called trypophobia (Abbasi, 2011; Cole \& Wilkins, 2013). It increasingly garnered public attention via the Internet and has then been investigated since the 2010s (Abbasi, 2011; Cole \& Wilkins, 2013). Accordingly, it seems to be a relatively new emotional phenomenon. Previous studies have revealed the relationship between trypophobia and visual processing. Cole and Wilkins (2013) performed a spectral analysis of trypophobic images (e.g., lotus seed pod) and neutral images (e.g., golf cup) and showed that these images had high contrast energy at midrange spatial frequency in comparison to the neutral images. Their study indicated that spatial frequency information at midrange was involved with trypophobia. Another study revealed that low- and mid-spatial frequency information contributed to trypophobia (Sasaki, Yamada, Kuroki, \& Miura, 2017). Based on these findings, trypophobic objects may be processed rapidly and unconsciously; indeed, there is empirical evidence supporting this (Sasaki, Watanabe, \& Yamada, 2018; Shirai \& Ogawa, 2019). Taken together, early visual processing plays key roles in trypophobia. trypophobia? Several studies have discussed cognitive factors of trypophobia. Recent studies have developed the Trypophobia Questionnaire (TQ) and investigated the relationship between trypophobia and personal traits (Chaya, Xue, Uto, Yao, \& Yamada, 2016; Le, Cole, \& Wilkins, 2015; Imaizumi, Furuno, Hibino, \& Koyama, 2016a). In particular, Imaizumi and his colleagues showed that core disgust (i.e., emotional avoidance of pathogen infection) sensitivity and positively predicted the TQ score (Imaizumi, Furuno, Hibino, \& Koyama, 2016b). This finding indicates that disgust contributes to trypophobia, and other findings also support this idea (Kupfer 
60 \& Le, 2018; Vlok-Barnard \& Stein, 2017). Based on these studies, Yamada and Sasaki (2017)

61 proposed the "involuntary protection against dermatosis" (IPAD) hypothesis. According to this

62 hypothesis, trypophobic objects evoke unpleasant emotions because their appearance is

63 associated with dermatosis and, as a result, an avoidance reaction to pathogens is induced. Indeed,

64 Yamada and Sasaki also provided evidence supporting the IPAD hypothesis; the history of skin

65 problems involves trypophobic discomfort. Thus, disgust toward infectious pathogens should 66 involve trypophobia.

Studies on trypophobia have rapidly increased, as we mentioned above. However, these studies were mainly conducted in the West and in Japan. Additionally, numerous cross-cultural studies on emotions show cultural differences in emotional processing (Grossmann, Ellsworth, \& Hong, 2012; Grossmann, Karasawa, Kan, \& Kitayama, 2014; Hot, Saito, Mandai, Kobayashi, \& Sequeira, 2006; Kitayama, Mesquita, \& Karasawa, 2006; Masuda, Ellsworth, Mesquita, Leu, Tanida, \& Van de Veerdonk, 2008; Tanaka, Koizumi, Imai, Hiramatsu, Hiramoto, \& de Gelder, 2010), although these studies mainly addressed general positive and negative emotions. Recently, given technological advances, it has become possible for behavioral data to be collected from less-urbanized areas, which was previously difficult (Takahashi, Oishi, \& Shimada, 2018). Considering that trypophobia has become popular and been studied since about 2010, mainly in

Western and Japanese culture areas (Abbasi, 2011; Cole \& Wilkins, 2013), it might be a modern emotion that possibly involves urbanization, and people in less-urbanized areas might not experience trypophobia. To address this issue, we examined whether trypophobia occurs in lessurbanized areas in China.

In the current study, we focused on two regions in Southwest China, Yunnan Province 82 and Guangxi Zhuang Autonomous Region. Yunnan province has mountains at its southwestern 
83

84

85

and southern part, including the Ailao and Wuliang Mountains along the southern coast of the Honghe River. In these mountainous areas, several ethnic minorities live a less-urbanized life. For example, Hani people, a mountainous ethnic group in the frontier of the country, are distributed along the Ailao and Wuliang Mountains. Dai people also live in the Honghe region, but are more concentrated in valleys or the relatively flat regions of the Honghe River basin. Guangxi Zhuang Autonomous Region is located at the southeast edge of the Yunnan-Guizhou Plateau. Yao people live in rural areas of the northwest and northeast mountainous and hilly areas. Thus, the present study examined whether people in the less urbanized areas of the Honghe region of Yunnan (i.e., Hani and Dai people) and Qibainong mountainous area in northwest of Guangxi (i.e., Yao people) experience trypophobia. As the control group, we also asked Chinese people in an urbanized area in the Yunnan Province, China and in Fukuoka City, Japan, if they experienced trypophobia. We hypothesized that trypophobia would be more salient as urbanization progressed. Therefore, we predicted that less urban people would experience weaker discomfort from the trypophobic images than would urban people and that, in less urban people, there would be no or small difference in the degree of discomfort between the neutral and trypophobic images.

\section{Ethics statement}

The present study received approval from the psychological research ethics committee of the Faculty of Human-Environment Studies at Kyushu University (approval number: 2018-002). The experiment was conducted according to the guidelines laid down in the Helsinki declaration. Informed consent was verbally obtained from all participants because some were pre-literate. 
105 Participants had the right to withdraw from the experiment at any time without providing any

106 reason.

107 Participants

108 We performed a two-way mixed-design analysis of variance (ANOVA) with the image

109 type (trypophobic and neutral) as a within-participant factor and the participant group (less urban

110 and urban people) as a between-participant factor. The required sample sizes were calculated

111 using G*power (Faul, Erdfelder, Lang, \& Buchner, 2007). We mainly intended to test whether

112 there is an interaction effect between the image type and the participant group. Thus, we

113 performed a preliminary test to detect the interaction effect $(\alpha=.05,1-\beta=.80, f=.25)$, and then

114 estimated the sample size to be 34 (i.e., 17 participants per exposure condition). However, a

115 previous study suggests that at least 20 participants are required per group to avoid Type I errors

116 (Simmons, Nelson, \& Simonsohn, 2011). Thus, we set the minimal sample size to 40 participants

117 (i.e., 20 per exposure condition) for the statistical analysis.

118 Thirty-four ethnic minorities living in less-urbanized areas (21 males and 13 females,

119 mean age $\pm \mathrm{SEM}=48.9 \pm 3.34$ years $)$ and 34 students in urban areas (11 males and 23 females,

120 mean age $\pm \mathrm{SEM}=23.6 \pm 0.36$ years) participated in the experiment ${ }^{1}$. The ethnic minorities of

121 the less urban group consisted of 10 Hani, 10 Dai, and 14 Yao people. Dai participants' data were

122 collected from the Qimaba town of the Huayao Dai minorities, while those of Hani participants

123 were collected from Hani villages around Lüchun County. Both fields were in Honghe Hani and

124 Yi Autonomous Prefecture of Yunnan Province, China. We also collected data of Yao people

125 from the Qibainong Area of the Yao villages in Guangxi Zhuang Autonomous Region, China.

126 The data of urban students were collected from the campus of Yunnan University in Kunming,

127 China and Kyushu University in Fukuoka, Japan. This urban group consisted of Han (the ethnic 
128 majority in China), Yi, Jingpo, and Zhuang peoples. Though the last three are relative minority 129 groups among Yunnan and Kyushu University students, they are sufficient to be regarded as a 130 control group because they had lived in a city for a long time and were urbanized to a large extent.

131 All of them were naive to the purpose of this experiment and reported having normal vision. Apparatus and stimuli

The stimuli were presented on a laptop (Dell Inspiron 7460). The resolution was $1920 \times$

1341080 pixels, and the refresh rate was $100 \mathrm{~Hz}$. The presentation of the stimuli and the collection of data were controlled by a computer. The stimuli were generated using PsychoPy3 (Peirce, 2007) and included 20 trypophobic and 20 neutral images $(512 \times 512$ pixels $)$ used in previous studies (Le et al., 2015; Sasaki et al., 2017; Yamada \& Sasaki, 2017).

138

\section{Procedure}

The participants initiated each trial by pressing the spacebar on a computer keyboard. After the fixation mark was presented for $500 \mathrm{~ms}$, the image stimulus appeared. They had to erase the stimulus by pressing a key when they thought that they had observed the stimulus enough. The rating scales were then presented. The participants were asked to evaluate the degree of discomfort for each image on a nine-point scale that ranged from 1 (strong discomfort) to 9 (strong comfort). Each participant performed 40 trials: two image types (trypophobic and neutral) $\times 20$ images. Trials were randomized for each participant. Afterward, a post-experimental interview was performed. The interview began with the question of whether participants felt uncomfortable when they saw images of a single hole or clusters of holes. If they answered "Yes," the subsequent question was about the part that made them feel uncomfortable. If they answered "No," the subsequent question was about the feeling they actually experienced when they looked at these pictures and why they felt it. 
151

154

155

156

157

158

159

160

161

162

163

164

165

166

167

168

169

170

171

172

173

\section{Analysis}

We calculated the average rating scores of the trypophobic and neutral images for each participant. We performed a two-way mixed ANOVA on the rating score with the image type as a within-participant factor and the participant group as a between-participant factor. When the interaction between the image type and participant group was significant, we performed a test of the simple main effects. We set the significance level at $\alpha=.05$ and reported $\eta_{p}{ }^{2} \mathrm{~s}$.

\section{Results}

The results are shown in Figure 1. The ANOVA revealed that the main effects of the image type and the participant group were significant (image type: $F(1,66)=106.31, p<.001, \eta_{p}{ }^{2}$ $=.62$; participant group: $F(1,66)=8.92, p=.004, \eta_{p}^{2}=.12$ ). Moreover, the interaction was significant $\left(F(1,66)=31.04, p<.001, \eta_{p}{ }^{2}=.32\right)$. The simple main effect of the image type was significant in both the participant groups (less urban people: $F(1,33)=16.05, p<.001, \eta_{p}{ }^{2}=.33$; urban people: $\left.F(1,33)=96.99, p<.001, \eta_{p}{ }^{2}=.75\right)$. Furthermore, the simple main effect of the participant group was significant for the trypophobic image $\left(F(1,66)=19.29, p<.001, \eta_{p}{ }^{2}=.23\right)$ while it was not significant in the neutral image $\left(F(1,66)=0.24, p=.63, \eta_{p}^{2}=.004\right)^{2}$.

After the experiment, participants verbally reported their impressions. Except for simple answers such as "yes" or "uncomfortable" to the questions about whether participants felt uncomfortable, a part of the other detailed reports are shown in Table 1 . The full interview reports are available at the data repository (https://osf.io/wvu8z/). --Figure 1 and Table 1 around here-- 


\section{Discussion}

175

The present study demonstrated that trypophobia occurs in Chinese ethnic minorities in

176 less urban as well as urbanized people. As mentioned above, we hypothesized that urbanization

177 is one of the factors contributing to trypophobia, and to examine this hypothesis, a comparison

178 of the effect sizes of trypophobia on less urban and urban people would be informative. Based on

179 the results for each group, a smaller effect size was found for less urban $\left(\eta_{p}^{2}=.33\right)$ than for urban

180 people $\left(\eta_{p}^{2}=.75\right)$. Briefly, trypophobia was salient in urban people in comparison with less urban

181 people. Additionally, the rating score of the trypophobic image was significantly lower in urban

182 than in less urban people, while there was no significant difference in the neutral image scores.

183 Thus, the urban people experienced more discomfort from the trypophobic, but not neutral,

184 images than did the less urban people. These results suggest that trypophobia comes from 185 urbanization.

Does urbanization alone regulate trypophobic responses? This may not be true. There are several differences in attributes and living environments between less urban and urban people; one possibility is a difference in age. In actuality, the participants' age was significantly higher in the less urban than in the urban people (Welch's $t$-test: $t(33.75)=7.54, p<.001$, Cohen's $d=$ 1.83). Did the participants' age also link to trypophobia? According to the post-experimental interview, the older less urban people do not experience much trypophobia. Moreover, the posthoc tests showed that the differences in the rating scores (i.e., subtracting the scores of the trypophobic image from those of the neutral ones) significantly correlated with the participants' age $(r=-.37, p=.002)$, indicating that trypophobia weakened with participants' higher age. A similar (but considerably weak) pattern was found in a previous study (Imaizumi et al., 2016a), which used the TQ with Japanese people. Taken together, although we could not clearly conclude 
197 which factor contingent to urbanization involves trypophobia, age-related properties are possibly

198 crucial ones.

The most interesting result of the present study was that, as mentioned earlier, even people

200 living in less-urbanized regions strongly experienced trypophobia, suggesting its high prevalence.

201 Here, one question arises: since when did this phenomenon exist? The study of trypophobia has

202 been rapidly increasing since 2000. To the best of our knowledge, the oldest case of a fear of a

203 hole, which seems to be very similar to trypophobia, was described in 1998 (Rufo, 1998). Our

204 urbanization hypothesis can help to answer this question: trypophobia is a phenomenon that

205 became stronger with urbanization, so it is likely that it was not strong enough to be discovered

206 until global urbanization fully progressed. Furthermore, the rapid spread of trypophobia may be

207 related to the development of image processing and information communication technologies

208 (e.g., the Internet): someone experienced trypophobia, then posted this experience on a website

209 (e.g., blog or forum), and as a result, trypophobia spread over the world ${ }^{3}$. These occurrences

210 played a critical role in the creation, processing, and dissemination of trypophobic images. In

211 addition, because of the digital divide (Norris, 2001), the development produced a gap in

212 familiarity with information technology between regions and generations, which supports our

213 urbanized-trypophobia hypothesis: if there is any positive correlation between age and

214 trypophobia intensity, this may be explained by the digital divide.

215 Because of differences in hygienic conditions, the severity of infectious diseases is

216 significantly higher in less-urban regions than in urban areas (Paddock, 2014; World Health

217 Organization, 2002). Are these difference in the severity of infectious diseases associated with

218 the present findings? Based on the IPAD hypothesis (Yamada \& Sasaki, 2017), trypophobia is

219 assumed to be an avoidance reaction to infectious pathogens because the appearance of 
220 trypophobic images is associated with skin diseases. This theory would predict that people in

221 less-urban areas would experience trypophobia more strongly than would people in urban areas;

222 however, this was not the case. Therefore, the difference in the severity of infectious diseases

223 cannot simply account for the difference in trypophobia between urban and less-urban areas. The

224 present findings give rise to a new possibility: infection habituation. People in less-urban areas

225 are frequently in contact with infectious pathogens (Paddock, 2014; World Health Organization,

226 2002) and as a result, are habituated to them. Such habituation might reduce the fear of infectious

227 diseases and, in turn, weaken the avoidance response to apparent sources of infection, thereby

228 attenuating trypophobia. Further experimentation on this point is thus warranted.

The present study has some limitations worth discussing. First, our experiment was conducted using a laptop computer, but the middle-aged and older people in less-urban area were embarrassed about their performances using such digital experimental device. The difficulty in operation possibly caused increased variance in the data. Moreover, the embarrassment may have somewhat contaminated and biased the emotional responses of comfort in the minority sample, although the neutral condition did not differ between the groups. Second, less-urban participants included those who did not understand Mandarin Chinese, and hence, the experimental instruction sometimes required translation by experimentally naive local guides who used local ethnic languages. This translation may have changed nuances, affecting the results. In future research, these problems may be solved through non-digital methods such as paper experiments and by inviting local psychological scientists to conduct experiments. Third, there were ethnic differences between urbanized and less-urbanized areas and these differences might have potential effects on our results that have not yet been discovered. Although there is no reasonable hypothesis on the differences in trypophobia among ethnic groups at this time and thus it is less 
243 likely that the ethnic differences contaminated our results, controlling these differences might be 244 desirable in future studies.

Fourth, all participants reported that their visual acuity was normal, but their visual

246 function was not actually measured and could not be confirmed. This may also affect the

247 evaluation results. Indeed, provocative visual patterns in the modern urban environments possibly

248 involve visual stress (Wilkins, Penacchio, \& Leonards, 2018), which is linked to trypophobia

249 (Imaizumi et al., 2016b). Moreover, as Sasaki and colleagues (2017) revealed, trypophobia is a

250 phenomenon that is dependent on spatial frequency. According to their study, high-frequency

251 components only minorly contributed to the strength of trypophobia. Differences in visual

252 function have often been argued about in studies on cultural differences in visual perception (e.g.,

253 Ahluwalia, 1978; Berry, Poortinga, Breugelmans, Chasiotis, \& Sam, 2012; Jahoda, 1971). If less-

254 urban participants who participated in this study had visual characteristics biased toward

255 processing of high-frequency components, it is possible that trypophobia as seen in the present 256 results is underestimated.

Last but not least, through this research in the Honghe and Qibainong Areas, we found that the development of trypophobia in less-urban areas, which had been experimented with previously, greatly exceeded the initial expectations. These areas can still have some unique

260 cultural symbols of ethnic minorities, such as residents wearing traditional ethnic costumes and 261 holding traditional ceremonies during festivals. However, in recent times, the wooden buildings 262 have been transformed into brick buildings and most residents skillfully use mobile phones and 263 electronic payments. These phenomena show that urbanization and modernization are accelerating even in the minority areas along the border. Therefore, the boundaries between urban and less-urban areas in China are gradually dissolving. Nevertheless, the results of the present 
266 study also seem to tell us that even in the course of such transformations, the regional differences

267 in terms of trypophobia still exist. Thus, testing trypophobia in some more primitive ethnic

268 settlements, such as with the Dulong in Nujiang, Yunnan, or ethnic minority areas in Thailand,

269 Laos, and Myanmar, concerning the difference between these peripheral regions and urban areas

270 is worth exploring in the future.

271

272

Conclusions

273 The present study aimed at testing whether trypophobia occurs in people in rural areas,

274 especially in Chinese ethnic minorities. Our hypothesis was that people in rural areas

275 experienced weaker trypophobia than people in urban areas. Consistent with this hypothesis,

276 weaker trypophobia occurred in rural people, suggesting that trypophobia is a relatively new

277 emotion that has emerged through urbanization. Future research using more peoples secluded

278 from modern civilizations is needed.

279

Peer) reviewing PDF | (2019:10:42574:3:0:NEW 18 Feb 2020) 


\section{References}

281

282

283

284

285

286

287

288

289

290

291

292

293

294

295

296

297

298

299

300

Abbasi, J. (2011, July 25). Is trypophobia a real phobia? Popular Science. Retrieved from http: //www.popsci.com/trypophobia

Ahluwalia, A. (1978). An intra-cultural investigation of susceptibility to 'perspective' and 'nonperspective' spatial illusions. British Journal of Psychology, 69(2), 233-241.

Berry, J. W., Poortinga, Y. H., Breugelmans, S. M., Chasiotis, A., \& Sam, D. L. (2012). Crosscultural psychology: Research and applications. Cambridge: Cambridge University Press. Chicago.

Chaya, K., Xue, Y., Uto, Y., Yao, Q., \& Yamada, Y. (2016). Fear of eyes: Triadic relation among social anxiety, trypophobia, and dis- comfort for eye cluster. PeerJ, 4:e1942.

Cole, G. G., \& Wilkins, A. J. (2013). Fear of holes. Psychological Science, 24, 1980-1985.

Faul, F., Erdfelder, E., Lang, A-G., \& Buchner, A. (2007). G*Power 3: a flexible statistical power analysis program for the social, behavioral, and biomedical sciences. Behavior Research Methods, 39, 175-191.

Grossmann, I., Ellsworth, P. C., \& Hong, Y. Y. (2012). Culture, attention, and emotion. Journal of Experimental Psychology: General, 141, 31-36.

Grossmann, I., Karasawa, M., Kan, C., \& Kitayama, S. (2014). A cultural perspective on emotional experiences across the life span. Emotion, 14, 679-692.

Hot, P., Saito, Y., Mandai, O., Kobayashi, T., \& Sequeira, H. (2006). An ERP investigation of emotional processing in European and Japanese individuals. Brain Research, 1122, 171-178. 
301 Imaizumi, S., Furuno, M., Hibino, H., \& Koyama, S. (2016a). Development of the Japanese 302 Version of Trypophobia Questionnaire. The Japanese Journal of Personality, 25, 171173. (in Japanese)

Imaizumi, S., Furuno, M., Hibino, H., \& Koyama, S. (2016b). Trypophobia is predicted by disgust sensitivity, empathic traits, and visual discomfort. SpringerPlus, 5, 1-5.

Jahoda, G. (1971). Retinal pigmentation, illusion susceptibility and space perception. International Journal of Psychology, 6(3), 199-207.

Kupfer, T. R., \& Le, A. T. (2018). Disgusting clusters: Trypophobia as an overgeneralised disease Methods, 162, 8-13. avoidance response. Cognition and Emotion, 32, 729-741.

Le, A. T., Cole, G. G., \& Wilkins, A. J. (2015). Assessment of trypophobia and an analysis of its visual precipitation. Quarterly Journal of Experimental Psychology, 68, 2304-2322.

Masuda, T., Ellsworth, P. C., Mesquita, B., Leu, J., Tanida, S., \& Van de Veerdonk, E. (2008). Placing the face in context: Cultural differences in the perception of facial emotion. Journal of Personality and Social Psychology, 94, 365-381.

Peirce, J. W. (2007). PsychoPy-psychophysics software in python. Journal of Neuroscience

Norris, P. (2001). Digital divide: Civic engagement, information poverty, and the Internet worldwide. Cambridge: Cambridge University Press. Chicago. 
322 Paddock, C. (2014, November 21). Water and sanitation in rural areas are 'seriously underfunded'.

323

324

325

326

327

328

330

331

332

333

334

335

336

337

338

339

340

341

342

Medical

News

Today.

Retrieved

from https://www.medicalnewstoday.com/articles/285848.php.

Rufo, M. (1998). La petite fille qui avait peur des trous. Soins Pédiatrie/Puériculture, 182, 3.

Sasaki, K., Watanabe, K., \& Yamada, Y. (2018). Invisible but unpleasant: Unconscious emotional processing of trypophobic objects. PsyArXiv, psyarxiv.com/5xshq

Sasaki, K., Yamada, Y., Kuroki, D., \& Miura, K. (2017). Trypophobic discomfort is spatial frequency dependent. Advances in Cognitive Psychology, 13, 224-231.

Shirai, R., \& Ogawa, H. (2019). Trypophobic images gain preferential access to early visual processes. Consciousness and Cognition, 67, 56-68.

Simmons, J. P., Nelson, L. D., \& Simonsohn, U. (2011). False-positive psychology: Undisclosed flexibility in data collection and analysis allows presenting anything as significant. Psychological Science, 22, 1359-1366.

Takahashi, K., Oishi, T., \& Shimada, M. (2017). Is smiling? Cross-cultural study on recognition of emoticon's emotion. Journal of Cross-Cultural Psychology, 48, 15781586.

Tanaka, A., Koizumi, A., Imai, H., Hiramatsu, S., Hiramoto, E., \& de Gelder, B. (2010). I feel your voice: Cultural differences in the multisensory perception of emotion. Psychological Science, 21, 1259-1262.

Vlok-Barnard, M., \& Stein, D. J. (2017). Trypophobia: An investigation of clinical features. Revista Brasileira de Psiquiatria, 39, 337-341. 
343 Wilkins, A.J. Penacchio, O. and Leonards, U. (2018). The built environment and its patterns: a view from the vision sciences. Journal of Sustainable Design and Applied Research, 6, 41-48.

346 World Health Organization. (2002). The world health report 2002: Reducing risks, promoting healthy life. World Health Organization.

348 Yamada, Y., \& Sasaki, K. (2017). Involuntary protection against dermatosis: A preliminary observation on trypophobia. BMC Research Notes, 10:658. 
352 This research was supported by JSPS KAKENHI (15H05709, 16H01866, 17H00875, 17H06342, 353 17J05236, 18H04199, 18K12015, and 19K14482).

354

355

356

357

358

359

360

361

362

363

364

365

366

367

368

369

370

371

372

373

\section{7}

\section{Author Contributions}

Contributed to conception and design: ZS, KS, QK, YY

Contributed to acquisition of data: ZS, JY

Contributed to analysis and interpretation of data: ZS, KS, QK, YY

Drafted and/or revised the article: ZS, KS, JY, QK, YY

Approved the submitted version for publication: ZS, KS, JY, QK, YY

\section{Competing Interests}

The authors declare no competing financial interests.

4

Data Availability

The datasets are available at https://osf.io/wvu8z/

\section{Footnotes}

${ }^{1}$ Initially, 20 ethnic minorities living in less-urbanized areas and 22 students from Yunnan University (i.e., urban areas) participated in the experiment based on the power analysis. We obtained similar results to that of the main text. According to the reviewer's suggestion, we added additional data from 14 less urban people from Guangxi and 12 urban people from Kyushu University. 
3742 According to Reviewer 1's suggestion, we also performed a two-way mixed ANOVA on the

375 rating score with the image type as a within-participant factor and the participant's location

376 (Honge, Kunming, Qibainong, Qujing, and Fukuoka) as a between-participant factor to test

377 whether the variance due to the participant group (urban or less urban) was greater than that due

378 to the participant's location. As a result, both of the main effects and interaction were significant

379 (image type: $F(1,63)=59.83, p<.001, \eta_{p}^{2}=.49$; participant's location: $F(4,63)=3.78, p=$

$380.008, \eta_{p}{ }^{2}=.19$; interaction: $\left.F(1,63)=8.08, p=.004, \eta_{p}{ }^{2}=.34\right)$. The results suggest that the

381 variance due to the participant group $(F=8.92)$ was greater than that due to the participant's

382 location $(F=3.78)$.

$383 \quad{ }^{3}$ Reviewer 2 also experienced a similar case.

\section{Figure Legends}

387 Figure 1. The results of the experiment. The error bars indicate standard errors of the mean.

388 Table 1. Post-experimental interview reports. 
Table $\mathbf{1}$ (on next page)

Post-experimental interviews 


\begin{tabular}{|c|c|c|c|c|}
\hline Area & Ethnicity & Age & Gender & Report \\
\hline \multirow{10}{*}{ Less urban } & \multirow{6}{*}{ Dai } & 10 & Female & The porous images made me feel dizzy. \\
\hline & & 41 & Female & I felt that many holes were dirty. \\
\hline & & 40 & Female & I felt a little nauseous and scared of the porous images. \\
\hline & & 64 & Female & $\begin{array}{l}\text { No matter which image I saw, I felt good and did not feel dizzy or } \\
\text { uncomfortable. }\end{array}$ \\
\hline & & 72 & Male & $\begin{array}{l}\text { I did not feel uncomfortable. I evaluated the impression of the } \\
\text { images based on whether things inside the images were practically } \\
\text { useful or whether they were ornamentally valuable. }\end{array}$ \\
\hline & & 57 & Male & $\begin{array}{l}\text { It did not matter how many holes the images contained. If the } \\
\text { brightness of the image was dark, I felt uncomfortable. On the } \\
\text { other hand, its brightness was high, which made me comfortable. }\end{array}$ \\
\hline & \multirow{4}{*}{ Hani } & 42 & Female & $\begin{array}{l}\text { The images did not induce discomfort regardless of whether these } \\
\text { contained many holes. I prefer plaid clothes. }\end{array}$ \\
\hline & & 79 & Male & $\begin{array}{l}\text { I did not feel uncomfortable. I actually felt that the porous images } \\
\text { were better-looking. }\end{array}$ \\
\hline & & 30 & Female & $\begin{array}{l}\text { The image containing high-dense holes made me dizzy and thus I } \\
\text { did not want to look at them. }\end{array}$ \\
\hline & & 55 & Male & The images containing fewer holes were good. \\
\hline \multirow{11}{*}{ Urban } & \multirow{2}{*}{ Yi } & 25 & Female & I was very disgusted. \\
\hline & & 20 & Male & I felt nothing special about the images. \\
\hline & Jingpo & 25 & Female & $\begin{array}{l}\text { I hated the clusters of small holes, although the holes on the food were } \\
\text { good. }\end{array}$ \\
\hline & Han & 21 & Female & I did not feel good. \\
\hline & Han & 18 & Male & $\begin{array}{l}\text { The porous images were eye-catching, while I felt nothing special } \\
\text { about the other images. }\end{array}$ \\
\hline & Han & 23 & Female & $\begin{array}{l}\text { When I saw the porous image, I felt as if my scalp was numb and } \\
\text { my hair stood up. }\end{array}$ \\
\hline & Han & 26 & Male & $\begin{array}{l}\text { The holes would be disgusting if they were randomly arranged and } \\
\text { thus looked worm-eaten and like acne. However, if the holes were } \\
\text { regularly aligned like beehives, they were good. }\end{array}$ \\
\hline & Han & 22 & Female & $\begin{array}{l}\text { It did not matter if the porous images contained artificial objects. } \\
\text { However, they were disgusting if they contained too many holes. }\end{array}$ \\
\hline & Han & 23 & Male & $\begin{array}{l}\text { Holes on natural things made me nauseous. In particular, when } \\
\text { objects in the holes were similar to acne, I felt disgust. The dirty } \\
\text { images also made me nauseous. }\end{array}$ \\
\hline & Han & 23 & Male & When lots of holes were regularly aligned, they were disgusting. \\
\hline & Han & 24 & Female & $\begin{array}{l}\text { I felt nothing special about the images. When I saw the mushroom, } \\
\text { I even wanted to eat it. }\end{array}$ \\
\hline
\end{tabular}




\section{Figure 1}

Figure 1.

The results of the experiment. The error bars indicate standard errors of the mean. 


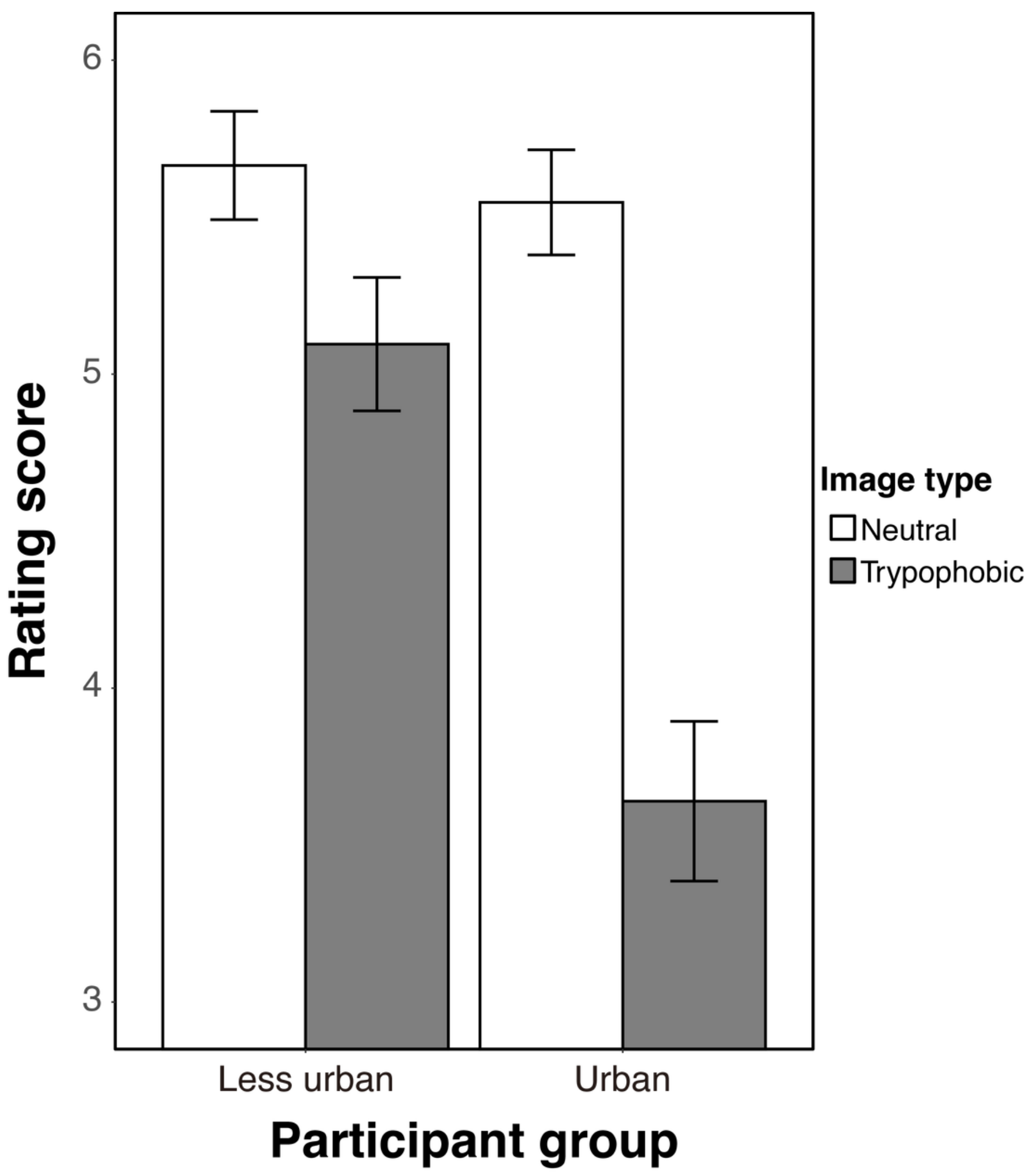

